Article

\title{
Effect of Hafnium Addition on the Hydrogenation Process of TiFe Alloy
}

\author{
Volatiana Razafindramanana ${ }^{1,2}$, Stéphane Gorsse ${ }^{1}\left[\begin{array}{l}\text {, Jacques Huot } \\ \text { 2,*(i) and Jean Louis Bobet }\end{array}\right.$ \\ 1 Univ. Bordeaux, CNRS, Bordeaux INP, ICMCB, UMR 5026, F-33600 Pessac, France \\ 2 Hydrogen Research Institute, Université du Québec à Trois-Rivières, 3351 boul., des Forges, C,P 500, \\ Trois-Rivières, QC G9A 5H7, Canada \\ * Correspondence: jacques.huot@uqtr.ca (J.H.); jean-louis.bobet@u-bordeaux.fr (J.L.B.)
}

Received: 23 June 2019; Accepted: 5 September 2019; Published: 9 September 2019

\begin{abstract}
The alloy TiFe has interesting hydrogen storage properties for practical applications: low cost, operation at room temperature, and good hydrogen capacity. However, the first hydrogenation is difficult and increases the cost of the alloy. In this work, we studied the effect of adding hafnium to TiFe in order to enhance the first hydrogenation process. TiFe $+\mathrm{x}$ Hf alloys, with $x=0,4,8,12$, and $16 \mathrm{wt} . \%$, were synthesized by arc melting. The microstructure of the as-cast alloys was investigated by scanning electron microscopy and electron microprobe analysis. These alloys consisted of B2-TiFe, C14-Laves, and BCC (Body Centered Cubic) phases. A minimum of $8 \mathrm{wt} . \%$ of hafnium is required to obtain an enhancement of the first hydrogenation. In the first hydrogenation, the material reaches its maximal hydrogen capacity in less than two hours at room temperature and under 20 bars of hydrogen. Hafnium addition also had the effect of lowering the plateau pressure in the pressure-composition isotherm. It could be concluded that hafnium has a positive effect on the activation properties of TiFe.
\end{abstract}

Keywords: TiFe alloy; hafnium addition; hydrogen storage; first hydrogenation

\section{Introduction}

Iron is one of the most abundant element in the Earth crust, and titanium is easily obtained at low cost. This makes the TiFe alloy relatively inexpensive and an auspicious material for solid-state hydrogen storage. This compound has been studied since the 70s and is now a well-known metal hydride [1]. Compared to other metals hydrides such as magnesium or body-centered cubic (BCC) alloys, its main advantage lies in the reversibility of hydrogen storage at room temperature. However, the first hydrogenation, the so-called activation process, is a lengthy and difficult operation because of the presence of a passivating oxidized surface. Therefore, activation needs to be performed at high temperature and high pressure [1,2]. In order to lower the production cost for industrial applications, it is mandatory to reduce these conditions. Several approaches have been used to enhance the first hydrogenation of TiFe. For example, partial substitution of Fe or Ti by $\mathrm{Al}, \mathrm{Mn}, \mathrm{Ni}$, or $\mathrm{Zr}$ could activate TiFe without any prior heat treatment [3-6]. An over stoichiometry of Ti could also facilitate the hydrogenation $[7,8]$. The addition of a dopant, such as $\mathrm{Pd}$ can be useful to reduce activation time $[9,10]$. More recently, several studies showed that forming a nanocrystalline TiFe compound at room temperature by mechanical alloying could improve the activation process [11-14]. Most of these studies demonstrated the importance of a third element and a strong relationship between the synthesis, the microstructure, and the hydrogen sorption of the TiFe alloy. A third element can also induce the formation of a secondary phase, which may enhance the hydrogen diffusion and its absorption in the material. Additionally, it could be incorporated in the TiFe crystal structure in solid solution.

Recently, it was found that adding only $4 \mathrm{wt} . \%$ of $\mathrm{Zr}_{7} \mathrm{Ni}_{10}$ to TiFe could greatly reduce the activation time under mild temperature and hydrogen pressure [15]. The alloy was found to be 
composed of a TiFe matrix phase and a secondary phase rich in zirconium. The effect of individual elements (i.e., zirconium and nickel) was investigated, and it was concluded that zirconium-rich phase plays an important role in the activation process improvement. Nickel has a negative effect on the hydrogen absorption properties compared to zirconium. Thus, the addition of only $\mathrm{Zr}$ has a positive effect on the activation of TiFe [16].

As industrial-grade zirconium always contains a small quantity of hafnium (Hf), it is of prime importance to study the role of Hf on the hydrogen sorption properties of TiFe. Our work focused on the understanding of the effect of $\mathrm{Hf}$ on the activation properties of TiFe, compared to zirconium. The microstructure and hydrogen sorption properties of TiFe doped with $\mathrm{xwt}$. $\% \mathrm{Hf}$, with $\mathrm{x}=0,4,8$, 12 , and 16 , synthesized by an arc melting furnace, are reported here.

\section{Materials and Methods}

Ternary TiFe-Hf alloys were synthesized by arc melting. For each sample, all the raw elements were weighed in the right proportion and melted all together. Each pellet was melted and turned over three times in order to get homogeneity in the alloys. The alloys were prepared under argon atmosphere to avoid any oxidation of the material.

Structural analysis was investigated by X-ray powder diffraction with a Bruker D8 Discover, with $\mathrm{Cu}-\mathrm{K}$ radiation. For $\mathrm{X}$-ray diffraction, the pellet was deduced into a powder using a mortar and pestle. Crystal structure parameters were extracted from the diffraction patterns by the Rietveld method using the TOPAS software. The microstructure was studied by a Scanning Electron Microscope, in backscattering mode in order to distinguish the different phases. Electron Probe MicroAnalysis (EPMA-CAMECA SX 100) determined the phase's composition and element mapping distribution. Finally, the first hydrogenation kinetic and pressure-composition-isotherms were measured using a homemade Sieverts-type apparatus. To perform the activation, each sample was crushed and reduced to a powder in a glove box, then exposed to hydrogen ( 20 bars) without any prior heat treatment.

Equilibrium and non-equilibrium calculations for the TiFe $+x \mathrm{Hf}$ alloys were performed using ThermoCalc (version 2018, Thermo-Calc software, Solna, Sweden) and the Thermo-Calc software TCHEA2 thermodynamic database. The Scheil-Gulliver model, which assumes infinite diffusion in liquid, and no diffusion in solid and local equilibrium condition at the liquid/solid interface, was applied to simulate the solidification path.

\section{Results}

This section may be divided by subheadings. It should provide a concise and precise description of the experimental results, their interpretation, as well as the experimental conclusions that could be drawn.

\subsection{Crystal Structure}

Figure 1 presents the X-ray powder diffraction patterns of TiFe with different Hf contents $(0,4$, $8,12$, and $16 \mathrm{wt.} \%)$ after solidification. Independently of the amount of hafnium, the main crystal structure is the B2-TiFe ( $\mathrm{CsCl}$ structure type; space group $\mathrm{Pm}-3 \mathrm{~m}$ ). However, a slight shift of the main peaks is observed with increasing Hf content.

Table 1 lists the lattice parameter and crystallite size for all compositions. There is a slight increase in the lattice parameters with Hf content. The crystallite size remains the same independent of hafnium content. For all the patterns, the microstrain was refined but found to be zero within experimental errors. When Hf content increases, $\mathrm{X}$-ray diffraction of the solidified alloys indicates the presence of a second phase with an $\mathrm{MgZn}_{2}$ hexagonal Laves structure (C14) of space group $\mathrm{P}_{3} / \mathrm{mmc}$. 


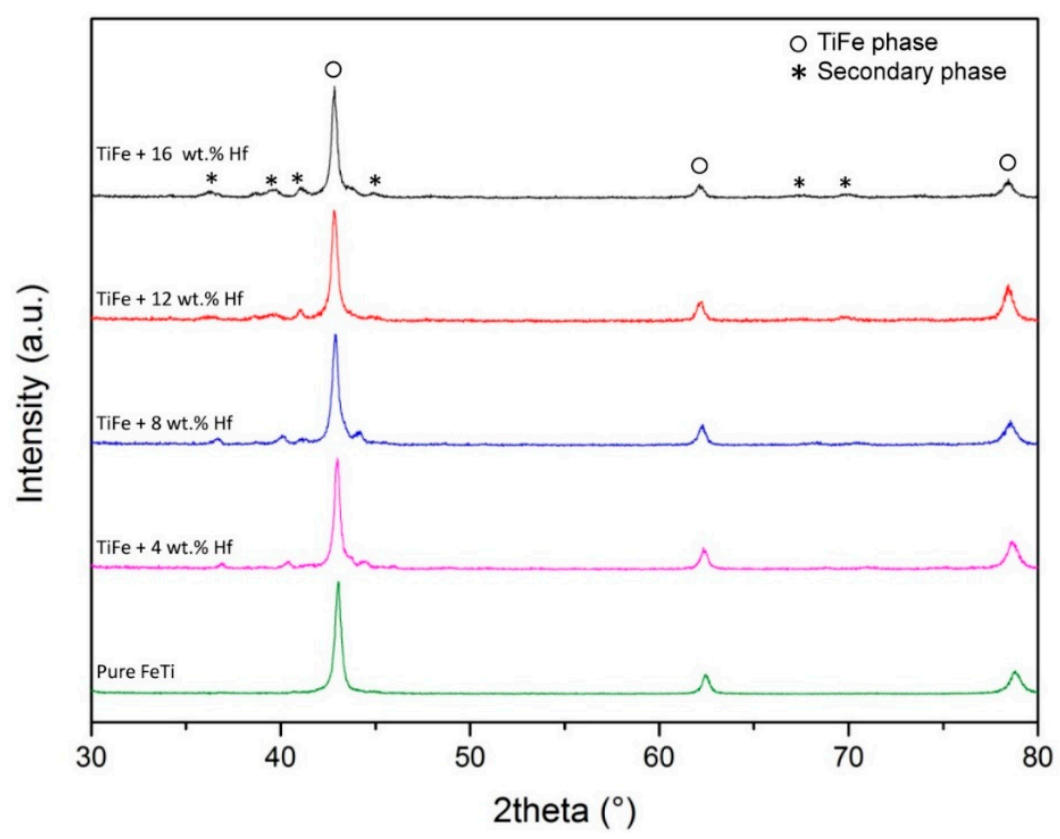

Figure 1. XRD (X Ray Diffraction) patterns of pure as-cast TiFe and TiFe $+x$ Hf alloys. $x=4,8,12$, and $16 \mathrm{wt} . \%$.

Table 1. Cell parameters and crystallites size of TiFe $+x$ wt. $\% \mathrm{Hf}(\mathrm{x}=0,4,8,12$, and 16). The uncertainty on the lattice parameters and crystallite sizes were identical for all composition and respectively $0.002 \AA$ and $1 \mathrm{~nm}$.

\begin{tabular}{cccc}
\hline $\mathbf{x}$ & $\mathbf{a}(\mathbf{A})$ & Crystallite Size (nm) & $\Delta \mathbf{\Delta a} / \mathbf{a}(\%)$ \\
\hline $\mathbf{0}$ & 2.977 & - & - \\
$\mathbf{4}$ & 2.982 & 17 & +0.17 \\
$\mathbf{8}$ & 2.986 & 15 & +0.30 \\
$\mathbf{1 2}$ & 2.989 & 17 & +0.40 \\
$\mathbf{1 6}$ & 2.990 & 17 & +0.44 \\
\hline
\end{tabular}

\subsection{Microstructure}

Figure 2. shows the typical dendritic microstructure of the TiFe $+x$ Hf alloys in the as-solidified condition. In the BSE (Back Scattered Electrons) mode, the dendrites are bright, and the inter-dendritic regions are dark, which reveals that dendrites have a higher mean atomic number, $\mathrm{Z}$, than the continuous phase. The fraction of dendrites increases with the amount of Hf. The higher magnification image reveals the presence of a third phase, with lighter $Z$ contrast, in the inter-dendritic regions.

The EDS elemental maps (Figure 3, Table 2) show that the dendrites are enriched with Fe, the inter-dendritic phase is primarily composed of $\mathrm{Ti}$ and Fe in equal amount, and the bright inter-dendritic particles are enriched with $\mathrm{Hf}$ and Ti. In conjunction with the elemental mapping, we also carried out composition measurements in several lines across the sample (60 $\mu \mathrm{m}$ in length) to more accurately determine the phase compositions. Each line was measured with several points. Table 2 presents the average phase atomic compositions for each sample (based on at least five points measured). 


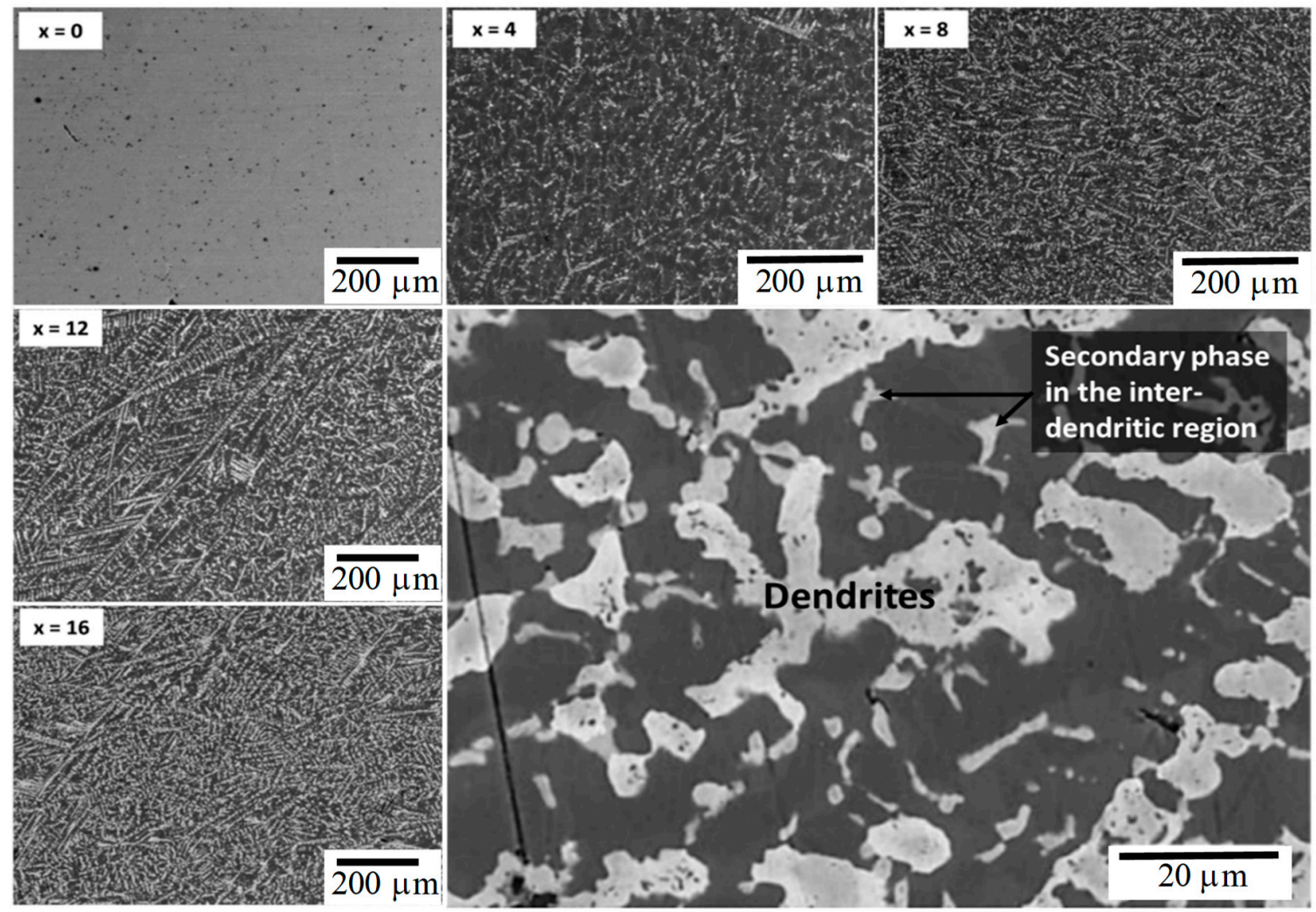

Figure 2. SEM (Scanning Electron Microscopy) images in backscattered (BSE) mode of the as-cast TiFe $+x$ Hf alloys (with $x=0,4,8,12$, and 16 wt. $\%$ ), and a higher magnification image of the TiFe +16 wt. $\%$ Hf alloy.

Table 2. Compositions (in at.\%) of the three phases in TiFe $+x$ Hf alloys (with $\mathrm{x}=4,8$ and 12, and 16 wt.\% of Hf) after solidification. Experimental data.

\begin{tabular}{cccc}
\hline (A) MATRIX (B2) & Ti & Fe & Hf \\
\hline $\mathbf{x}=\mathbf{4}$ & $49.9(0.9)$ & $49(1)$ & $1.5(0.6)$ \\
$\mathbf{x}=\mathbf{8}$ & $49.9(0.8)$ & $48.6(0.8)$ & $1.4(0.5)$ \\
$\mathbf{x}=\mathbf{1 2}$ & $49.6(0.9)$ & $48(1)$ & $2.4(0.5)$ \\
$\mathbf{x}=\mathbf{1 6}$ & $49.7(0.9)$ & $48(1)$ & $2.1(0.6)$ \\
\hline (B) Dendrite (C14-Laves) & $\mathbf{T i}$ & $\mathbf{F e}$ & $\mathbf{H f}$ \\
\hline $\mathbf{x}=\mathbf{4}$ & $40.5(0.8)$ & $55.8(0.9)$ & $3.6(0.6)$ \\
$\mathbf{x}=\mathbf{8}$ & $40(1)$ & $54(1)$ & $6.1(0.9)$ \\
$\mathbf{x}=\mathbf{1 2}$ & $41(2)$ & $52(2)$ & $8(2)$ \\
$\mathbf{x}=\mathbf{1 6}$ & $40(2)$ & $51(2)$ & $9(1)$ \\
\hline (C) Intergranular & $\mathbf{T i}$ & $\mathbf{F e}$ & $\mathbf{H f}$ \\
\hline $\mathbf{x}=\mathbf{4}$ & $57(1)$ & $35(1)$ & $7.9(0.7)$ \\
$\mathbf{x}=\mathbf{8}$ & $53.4 *(0.6)$ & $39.6 *(0.3)$ & $7.0 *(0.3)$ \\
$\mathbf{x}=\mathbf{1 2}$ & $51(2)$ & $37(2)$ & $12(2)$ \\
$\mathbf{x}=\mathbf{1 6}$ & $52.8(0.8)$ & $37(2)$ & $10(2)$ \\
\hline
\end{tabular}

* based on 2 points. 


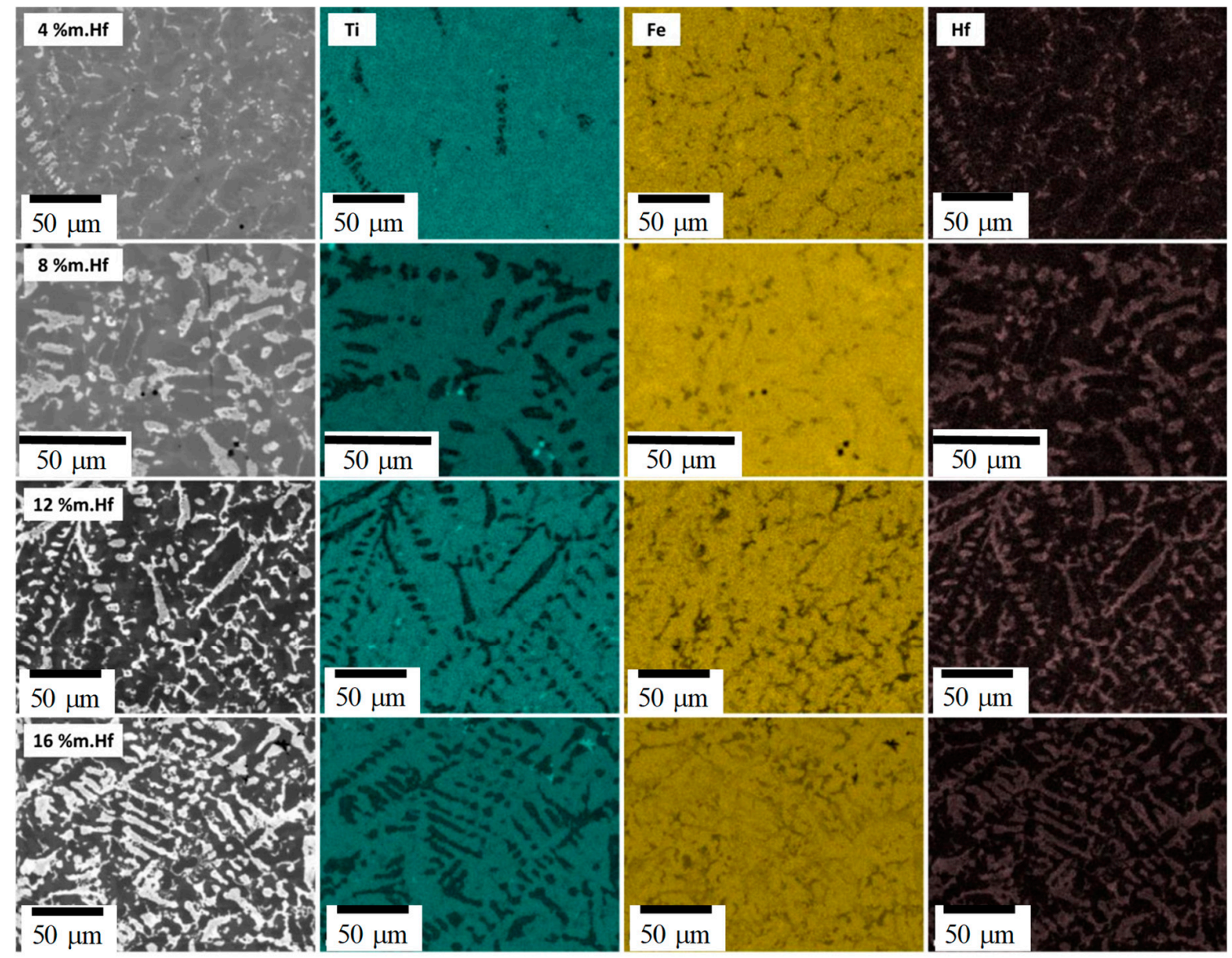

Figure 3. BSE images of the TiFe $+x$ Hf alloys (with $\mathrm{x}=0,4,8,12$, and 16 wt. $\%$ ) and EDS (Energy Dispersive Spectra) elemental maps of Ti, Fe, and Hf. Bright colors indicate a high content of the element, dark areas mean low abundance.

The composition of the dark phase is mainly the same for all samples. This dark phase corresponds to the TiFe matrix with a ratio Ti:Fe very close to 1 . However, a small amount of hafnium, up to 2.4 at. $\%$, is detected. This small amount of $\mathrm{Hf}$ in the TiFe matrix explains the difference in cell parameters reported in Table 1. Regarding the dendritic phase, the composition is very different. A higher iron proportion (around 53 at.\%) is measured, contrary to the percentage of titanium (40 at.\%). The hafnium is also more present in this phase than in the matrix. In Figure 3, it is also possible to highlight some very few spots which could be attributed to unreacted titanium.

The very small variation (increase) of lattice parameters of the B2 matrix phase reported in Table 1 can be explained using Table 2. The metallic radius of Hf is larger than the one of both Fe and Ti (respectively 1.58, 1.27, and $1.46 \AA$ ). As seen in Table 2, the ratio Fe/Ti is always very close to 1 , and the variations are very limited. Moreover, the amount of $\mathrm{Hf}$ (which could substitute either iron or titanium site) is also very limited, considering that a 2 at.\% of Hf in FeTi leads to a lattice parameter of $2.991 \AA$ with a mixed occupancy. Therefore, the slight variation of the lattice parameter can be either attributed to the change of the $\mathrm{Fe} / \mathrm{Ti}$ ratio or to the $\mathrm{Hf}$ content. As both variations are limited, the lattice parameter variation is very small (less than $0.4 \%$ ).

The solidification path using the Scheil-Gulliver simulation was done to understand better the microstructure observed. Figure $4 \mathrm{a}, \mathrm{b}$ show the fraction and the composition of each phase as a function of temperature during solidification of TiFe $16 \mathrm{wt} . \% \mathrm{Hf}$. The simulation indicates that the primary solidified phase is the Laves-C14 phase followed by the solidification of the ordered B2 phase, which corroborates the experimental observations from $X$-ray diffraction. Finally, a eutectic reaction Liquid $\rightarrow \mathrm{B} 2+\mathrm{BCC}$ occurs. 


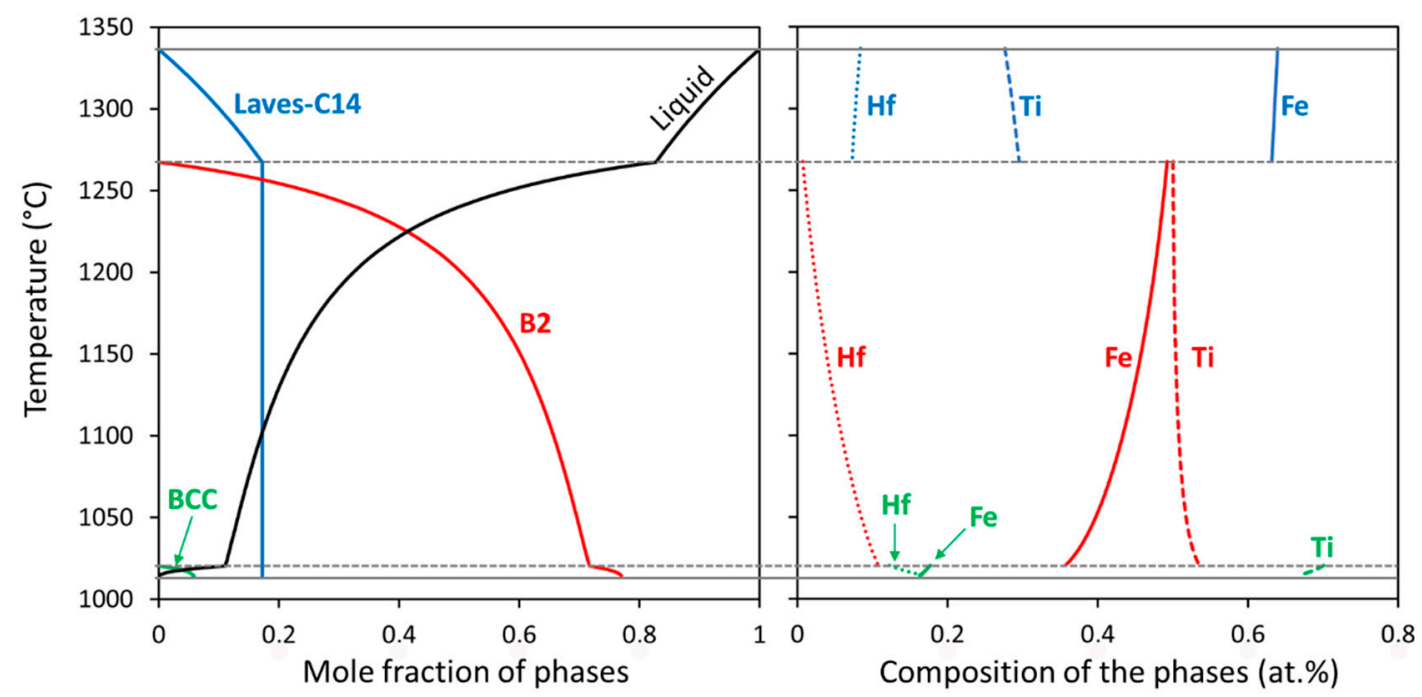

(a)

(b)

Figure 4. (a) Molar fractions and (b) composition (at.\%) of the phases (Liquid, Laves-C14, B2 and BCC) formed during non-equilibrium solidification of the TiFe 16 wt.\% Hf alloy.

According to this simulation, the alloy has three components: an $\mathrm{Fe}_{2} \mathrm{Ti}$ phase (Laves-C14) with Hf mixing mainly in the Ti sublattice, a B2 phase close to Ti/Fe equimolarity with a small amount of $\mathrm{Hf}$, and a BCC phase heavily enriched with Ti and containing a relatively large amount of Hf. A comparison of the simulation with the morphologies, spatial distributions, measured composition of the phases, and X-ray diffraction patterns indicates that the Fe-rich Laves phase solidified first in the form of dendrites, followed by the solidification of the B2-TiFe phase in the inter-dendritic regions, and the formation of the Ti-rich BCC phase during the last stages of solidification, possibly due to eutectic reaction. The solidification simulation supports the experimental observations (considering that the intergranular phase reported in Table 2 is a BCC) and explains the morphology of the microstructure. However, the observed and predicted compositions of the phase are slightly different.

The calculated FeTi $+x$ Hf isopleth (Figure 5) shows that a two-phase B2 + Laves-C14 field is present between 700 and $1150{ }^{\circ} \mathrm{C}$ for $\mathrm{x}<16 \mathrm{wt} . \%$. Therefore, the thermodynamic simulation suggests that the present as-solidified alloys, which consist of three phases, are not in the equilibrium condition.

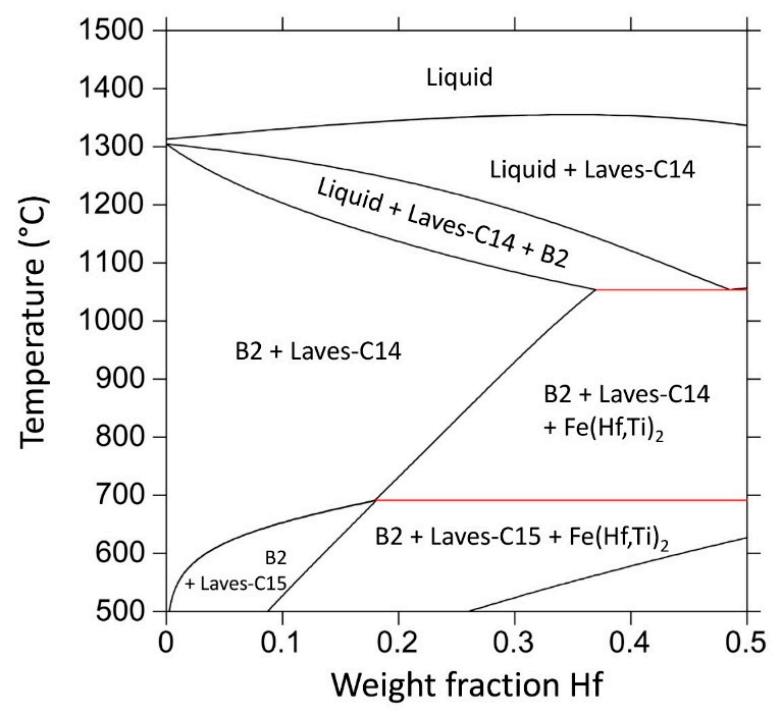

Figure 5. Calculated isopleths for TiFe $+x$ Hf (with $x=0-50 w t . \%$ ) showing predicted phases as a function of Hf content and temperature, and in particular the two-phase B2 + Laves-C14 field up to 37 wt. $\%$ Hf at $1055^{\circ} \mathrm{C}$. 


\subsection{Activation Characteristics}

Hydrogen absorption was done at room temperature, without any prior heat treatment. The effect of hafnium on the first hydrogenation is shown in Figure 6. As it can be seen, pure TiFe and TiFe + $4 \mathrm{wt} . \% 4 \mathrm{Hf}$ alloys do not absorb hydrogen even after 10 hours of hydrogen exposure. The hydrogen absorption behavior is drastically improved when the amount of added hafnium is higher than $8 \mathrm{wt} . \%$. There is no incubation time, the absorption occurs in a single step, and the hydrogen absorption kinetics increases with the amount of hafnium. The maximum capacity reaches $1.5 \%$ in less than $90 \mathrm{~min}$ for $16 \mathrm{wt} . \%$ Hf. The effect of Hf on the hydrogen absorption behavior of TiFe can be interpreted as due to the presence of the Laves phase. This kinetic improvement is comparable with the one obtained using zirconium as a dopant [16].

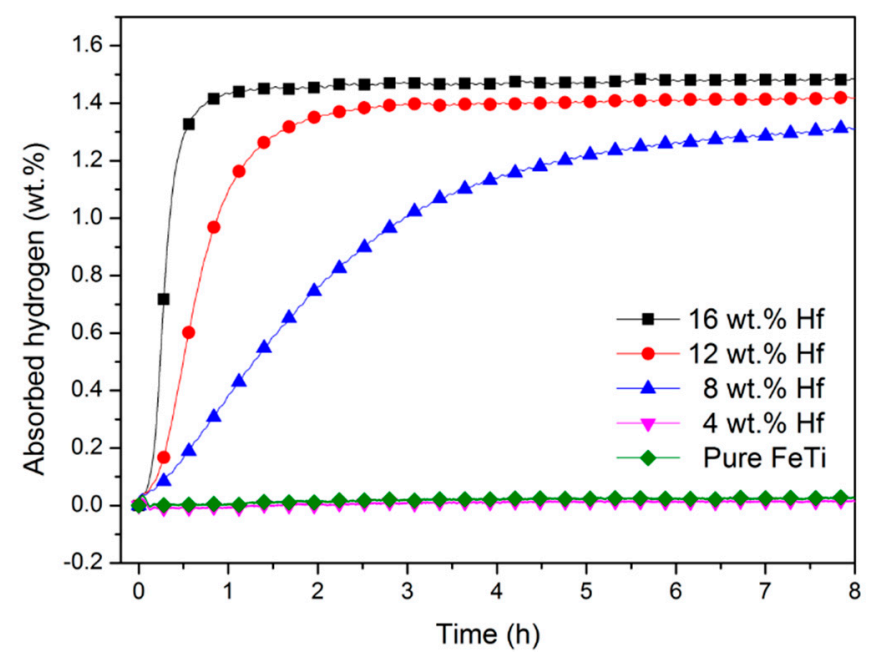

Figure 6. First hydrogenation curves of pure TiFe and TiFe doped with 4, 8, 12, and 16 wt.\% of Hf.

Measurements made at room temperature and under 20 bars of $\mathrm{H}_{2}$.

\subsection{Pressure-Composition-Isotherms}

Pressure-composition-isotherms were measured after the first hydrogenation at room temperature. Prior to the measurement, all the alloys were desorbed under dynamic vacuum at room temperature for $2 \mathrm{~h}$. Results are shown in Figure 7 for samples with $x=8,12$, and $16 \mathrm{wt}$.\% Hf. The behavior is quite the same for all alloys. It can be noticed that both the absorption and the desorption plateau pressure decrease with an increasing amount of hafnium. This effect has already been observed when zirconium is added as a dopant $[17,18]$. This means that the thermodynamics is slightly changed for a high amount of Hf.
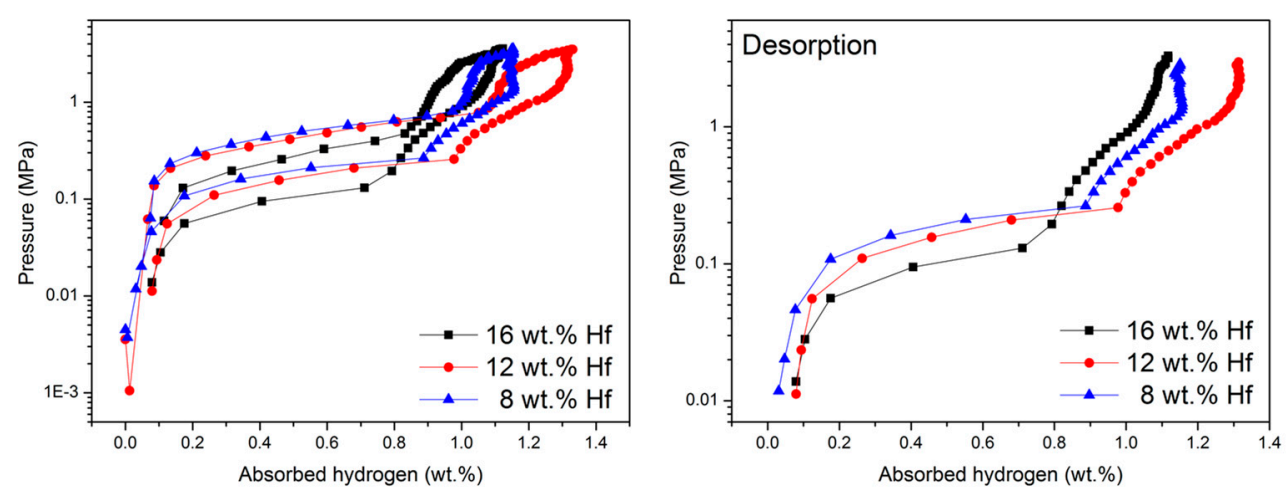

Figure 7. On the left, PCT (Pressure Composition Temperature) curves of TiFe $+8,12$, and 16 wt. $\%$ of $\mathrm{Hf}$; On the right, PCT desorption curves of all samples. Measurements were done at room temperature and between 0.5 and 30 bars of $\mathrm{H}_{2}$. 


\subsection{Cycling Effects}

Cycling measurements were done in the same experimental conditions as the first hydrogenation: room temperature and 20 bars of $\mathrm{H}_{2}$ for absorption and vacuum for desorption for at least three hours. Figure 8 shows the first hydrogenation and the next five absorption cycles. Two main observations could be highlighted:

(i) Kinetics becomes faster with cycling. From cycling 2, the maximum capacity is reached in less than $5 \mathrm{~min}$. This phenomenon is principally due to the creation of new clean surfaces caused by decrepitation occurring during hydrogenation. The new surfaces are propitious to be more sensitive to hydrogen absorption. Also, the hydrogen diffusion is greatly facilitated.

(ii) Decrease in the capacity. After the first cycle, the capacity reaches only $1.0 \%$ compared to $1.38 \%$ for the activation process. It can be concluded that after the first absorption, the alloy is fully activated and reversible under cycling. The difference between the capacity obtained after activation and after cycling may be due to the formation of a stable hydride created by the secondary phase. An image analysis performed from the EDS image (some reported in Figure 3) highlights that the average abundance of secondary phases is about $25 \%$. Then, considering such a hypothesis, the decrease of the sorption properties could be around 22\% (from 1.38 to 1.07). However, XRD post-hydrogenation analysis did not allow for highlighting the secondary hydride phase. Synchrotron and neutron experiments are underway in order to have more information about this secondary phase during hydrogenation and dehydrogenation.

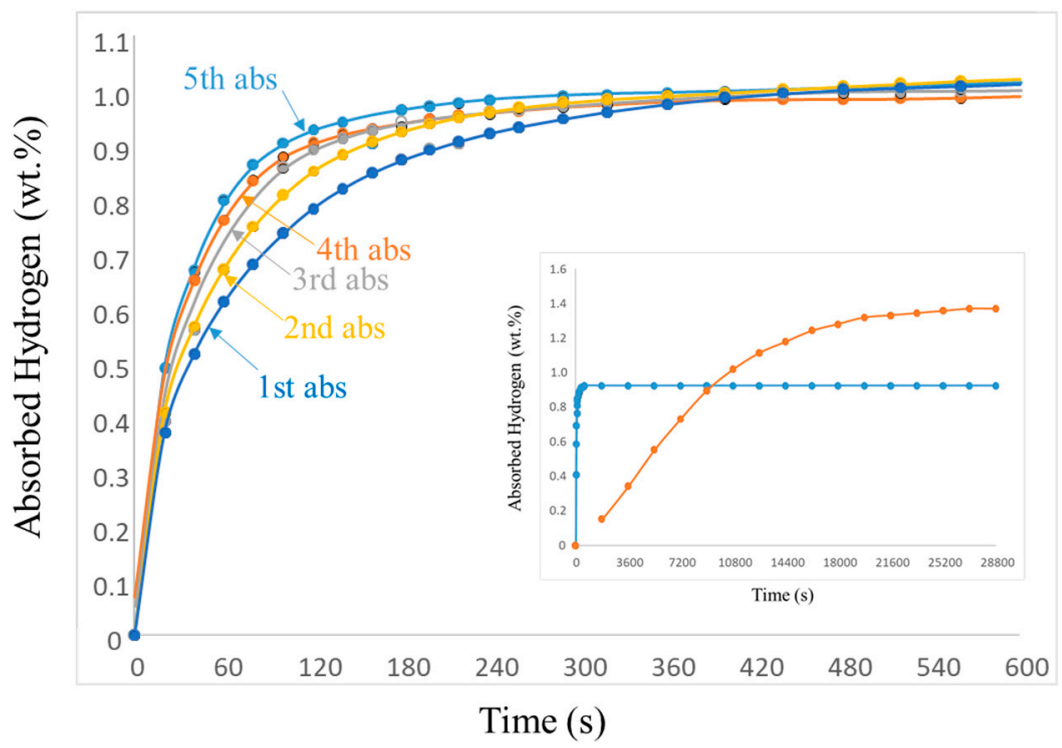

Figure 8. First hydrogenation (activation, in the inset) and cycling measurement of $\mathrm{TiFe}+12 \mathrm{wt} . \%$ of Hf. Measurements were done at room temperature and under 20 bars of $\mathrm{H}_{2}$.

\section{Conclusions}

The activation process of TiFe alloy could be enhanced without any heat treatment and without decreasing the hydrogen absorption capacity drastically by casting with the addition of at least $8 \mathrm{wt} . \%$ of hafnium. The presence of hafnium induces the formation of two secondary phases in addition to the main B2-TiFe phase: Fe-rich Laves-C14 and Ti-rich BCC. Independent of the quantity of hafnium added, the matrix composition remains almost the same; iron and titanium are in stoichiometric proportion with a low content of hafnium $(<2 \%)$ substituting for iron. Only $8 \mathrm{wt} . \%$ of $\mathrm{Hf}$ is necessary to activate the TiFe alloy, which is in good agreement with the result obtained for zirconium (i.e., more or less the same molar fraction; $4 \mathrm{wt} . \% \mathrm{Zr}=4 \mathrm{~mol} . \%$ and $8 \mathrm{wt} . \% \mathrm{Hf}=4.6 \mathrm{~mol} . \%$ ). The addition of hafnium results in the formation of two secondary phases which have a positive effect on the TiFe activation process. Also, adding hafnium reduces the plateau pressure slightly. Cycling improves 
the absorption kinetics but decreases the capacity. Therefore, trace amounts of hafnium present in commercial zirconium do not have a negative effect on the hydrogenation/dehydrogenation kinetics when it is used as a dopant. Unfortunately, the presence of a high proportion of the secondary phase drastically decreases the reversible hydrogen capacity.

Author Contributions: Conceptualization-J.H. and J.L.B.; Methodology-J.H. and J.L.B.; Validation-V.R.; Formal analysis-V.R., S.G., J.H. and J.L.B.; Investigation-V.R.; Writing-original draft preparation-V.R.; Writing-review and editing-J.H. and J.L.B.; Supervision-J.H. and J.L.B.

Funding: This research was funded by Région Aquitaine through a grant to V.R.

Conflicts of Interest: The authors declare no conflict of interest.

\section{References}

1. Reilly, J.J.; Wiswall, R.H. Formation and properties of iron titanium hydride. Inorg. Chem. 1974, 13, $218-222$. [CrossRef]

2. Schlapbach, L.; Riesterer, T. The activation of FeTi for hydrogen absorption. Appl. Phys. A 1983, 32, 169-182. [CrossRef]

3. Lim, S.H.; Jai-Young, L. The effects of aluminum substitution in TiFe on its hydrogen absorption properties. J. Less Common Metals 1984, 97, 65-71. [CrossRef]

4. Chung, H.S.; Lee, J.Y. Effect of partial substitution of Mn and Ni for Fe in FeTi on hydriding kinetics. Int. J. Hydrog. Energy 1986, 11, 335-339. [CrossRef]

5. Nagai, H.; Kitagaki, K.; Shoji, K. Microstructure and hydriding characteristics of FeTi alloys containing manganese. J. Less Common Metals 1987, 134, 275-286. [CrossRef]

6. Nishimiya, N.; Wada, T.; Matsumoto, A.; Tsutsumi, K. Hydriding characteristics of zirconium-substituted FeTi. J. Alloys Compd. 2000, 313, 53-58. [CrossRef]

7. Lee, S.-M.; Perng, T.-P. Microstructural correlations with the hydrogenation kinetics of FeTi 1 $_{1+x}$ alloys. J. Alloys Compd. 1991, 177, 107-118. [CrossRef]

8. Mizuno, T.; Morozumi, T. Titanium concentration in $\mathrm{FeTi}_{\mathrm{x}}(1<\mathrm{x}<2)$ alloys and its effect on hydrogen storage properties. J. Less Common Metals 1982, 84, 237-244.

9. Suzuki, R.; Ohno, J.; Gondoh, H. Effect of sulphur addition on the properties of FeTi alloy for hydrogen storage. J. Less Common Metals 1984, 104, 199-206. [CrossRef]

10. Zaluski, L.; Zaluska, A.; Tessier, P.; Strom-Olsen, J.O.; Schulz, R. Catalytic effect of Pd on hydrogen absorption in mechanically alloyed $\mathrm{Mg}_{2} \mathrm{Ni}_{1} \mathrm{LaNi}_{5}$ and FeTi. J. Alloys Compd. 1995, 217, 295. [CrossRef]

11. Aoyagi, H.; Aoki, K.; Masumoto, T. Effect of ball milling on hydrogen absorption properties of FeTi, $\mathrm{Mg}_{2} \mathrm{Ni}$ and $\mathrm{LaNi}_{5}$. J. Alloys Compd. 1995, 231, 804-809. [CrossRef]

12. Hotta, H.; Abe, M.; Kuji, T.; Uchida, H. Synthesis of Ti-Fe alloys by mechanical alloying. J. Alloys Compd. 2007, 439, 221-226. [CrossRef]

13. Emami, H.; Edalati, K.; Matsuda, J.; Akiba, E.; Horita, Z. Hydrogen storage performance of TiFe after processing by ball milling. Acta Mater. 2015, 88, 190-195. [CrossRef]

14. Abe, M.; Kuji, T. Hydrogen absorption of TiFe alloy synthesized by ball milling and post-annealing. J. Alloys Compd. 2007, 446-447, 200-203. [CrossRef]

15. Jain, P.; Gosselin, C.; Skryabina, N.; Fruchart, D.; Huot, J. Hydrogenation properties of TiFe with Zr7Ni10 alloy as additive. J. Alloys Compd. 2015, 636, 375-380. [CrossRef]

16. Jain, P.; Gosselin, C.; Huot, J. Effect of Zr, Ni and Zr7Ni10 alloy on hydrogen storage characteristics of TiFe alloy. Int. J. Hydrog. Energy 2015, 40, 16921-16927. [CrossRef]

17. Jang, T.H.; Han, J.I.; Lee, J.Y. Effect of substitution of titanium by zirconium in TiFe on hydrogenation properties. J. Less Common Metals 1986, 119, 237-246. [CrossRef]

18. Lv, P.; Huot, J. Hydrogen storage properties of Ti0.95FeZr0.05, TiFe0.95Zr0.05 and TiFeZr0.05 alloys. Int. J. Hydrog. Energy 2016, 41, 22128-22133. [CrossRef]

(C) 2019 by the authors. Licensee MDPI, Basel, Switzerland. This article is an open access article distributed under the terms and conditions of the Creative Commons Attribution (CC BY) license (http://creativecommons.org/licenses/by/4.0/). 